\title{
Low-threshold two-dimensional annular Bragg lasers
}

\author{
Jacob Scheuer \\ Department of Applied Physics, Mail Stop 128-95, California Institute of Technology, Pasadena, California 91125
}

William M. J. Green and Guy DeRose

Department of Electrical Engineering, Mail Stop 128-95, California Institute of Technology, Pasadena, California 91125

\begin{abstract}
Amnon Yariv
Departments of Applied Physics and Electrical Engineering, Mail Stop 128-95, California Institute of Technology, Pasadena, California 91125
\end{abstract}

\author{
Received July 29, 2004
}

\begin{abstract}
Lasing at telecommunication wavelengths from annular resonators employing radial Bragg reflectors is demonstrated at room temperature under pulsed optical pumping. Submilliwatt pump threshold levels are observed for resonators with $0.5-1.5$-wavelength-wide defects of radii 7-8 $\mu \mathrm{m}$. The quality factors of the resonator modal fields are estimated to be of the order of a few thousand. The electromagnetic field is shown to be guided by the defect. Good agreement is found between the measured and the calculated spectra. (C) 2004 Optical Society of America

OCIS codes: $140.4780,140.5960,250.3140,230.1480$.
\end{abstract}

Ring resonators are versatile elements with various applications ranging from telecommunication and sensing to basic scientific research. ${ }^{1-5}$ During the past few years, considerable effort has been focused on improving the quality factors $(Q)$ of resonators and reducing their modal volume (see, e.g., Refs. 5 and 6 and references therein).

Recently we proposed a novel type of annular resonator based on radial Bragg reflection. ${ }^{7,8}$ These devices, annular Bragg resonators (ABRs), offer smaller dimensions than those of conventional resonators that employ total internal reflection while retaining a high $Q$. In addition, such structures exhibit superior sensitivity compared with conventional resonators for biological and chemical sensing applications. ${ }^{9} \quad$ This class of resonators is closely related to the family of circulargrating distributed Bragg reflector resonators, which also exhibit lasing patterns with low angular propagation coefficients. ${ }^{10,11}$

In this Letter we report on the observation of photoluminescence and lasing from ABRs realized in semiconductor material (see Fig. 1). The structure consisted of a circumferentially guiding defect surrounded by radial Bragg reflectors. Because of the circular geometry, the optimal layer widths required to confine the light in the defect are not constant but rather monotonically decreasing with the radial distance. The widths of the layers are determined by the zeros and extrema of the Bessel function of order $m$, where $m$ is the angular propagation coefficient of the mode for which the device was designed. For simplicity we label the separation between a zero and the nearest extremum and between successive zeros the quarter-wavelength and the half-wavelength, respectively. Although these distances are not constant across the device, their role in the construction of the distributed reflector is similar to that of their Cartesian counterparts. ${ }^{12}$ The reflector layers could also be of higher Bragg order (i.e., $3 / 4 \lambda, 5 / 4 \lambda$, etc., in the same notation). Although such an approach requires more Bragg layers to confine the light in the defect (compared with a reflector based on $\lambda / 4$ layers), it facilitates the fabrication of the devices. The defect width of an ABR is a multiple integer of half-wavelength, meaning that its interfaces are located at zeros of the field.

ABRs of several geometries and Bragg reflector orders were fabricated within a 250 -nm-thick membrane of InGaAsP with six 7.5-nm quantum wells positioned at the center. After the ABR patterns were etched into the active material, the original InP substrate was removed and the membrane was transferred to a sapphire plate by use of an ultraviolet-curable optical adhesive to improve the vertical confinement of the electromagnetic field in the device. ${ }^{13}$

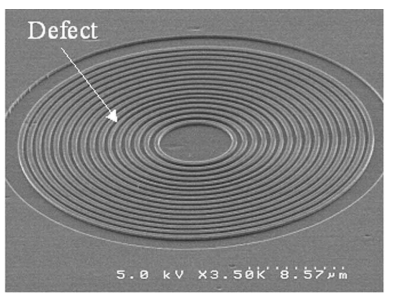

A

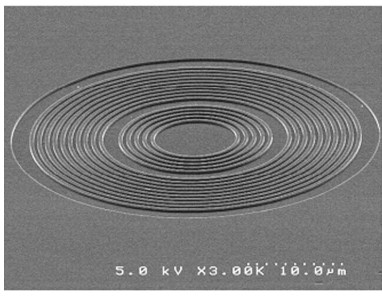

C

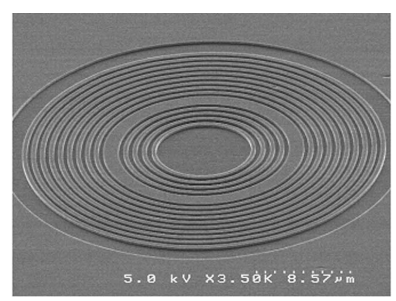

B

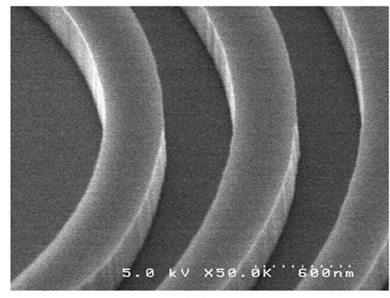

$\mathrm{D}$
Fig. 1. Scanning electron microscope images of the fabricated ABRs: A, $\lambda / 2$-wide high-index defect. The defect is the slightly narrower sixth ring from the center, marked

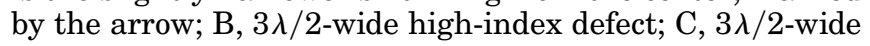
air defect; D, close up of the reflector layers. 
To simplify the design and the modeling of the devices we employed the effective index approximation in the vertical dimension. The effective index of the membrane was found to be approximately 2.8 for the $H_{z}$ polarization and 2.09 for the $E_{z}$ polarization. Since the $H_{z}$ polarization is more confined than the $E_{z}$ polarization and the optical gain of the compressively strained quantum-well structure used favors the $H_{z}$ polarization, ${ }^{14}$ we optimized the radial structure to this polarization. ${ }^{8}$

To facilitate the fabrication of the devices we adopted a mixed Bragg order approach. ${ }^{8}$ The highindex Bragg layers $\left(n_{\text {eff }}=2.8\right)$ were $3 / 4 \lambda$ wide (second order), and the low-index layers $\left(n_{\text {eff }}=1.0\right.$ for air gaps and $n_{\text {eff }}=1.54$ for adhesive-filled gaps) were $\lambda / 4$ wide (first order). In addition to the relaxed fabrication tolerances, such a layer structure improves the vertical confinement (larger material filling factor) and induces efficient vertical emission. Although the latter decreases the overall $Q$ factor of the cavity, it also permits simple observation of the intensity pattern that evolves in the device. ${ }^{15}$ Figure 1 depicts scanning electron microscope micrographs of some of the fabricated devices.

The near-field intensity pattern and the emitted spectrum of the ABRs were examined at room temperature under pulsed optical pumping. The pump beam was focused through the transparent sapphire substrate on the backside of the sample. Half of the pump beam intensity was split by a $3-\mathrm{dB}$ beam splitter and was focused on a detector to obtain the pump power. The vertical emission from the front side of the sample was either focused on an IR camera to obtain the near-field intensity pattern or coupled into a multimode fiber to obtain the spectrum.

The spectrum emitted from the pumped, unpatterned, quantum-well layer structure consisted of a wide peak centered at $1559 \mathrm{~nm}$. When an ABR was pumped, the emission characteristics changed significantly. Although the specific details varied from device to device, the overall behavior was similar. Once a certain pump intensity threshold was exceeded, clear and narrow emission lines appeared in the spectrum (see Fig. 2). As the pump intensity was increased, the intensity of the emission lines increased while broadening toward shorter wavelengths. Increasing the pump power further resulted in the appearance of additional emission lines.

Figure 2 depicts the emitted spectra from an ABR for various pumping levels. The specific device consisted of a half-wavelength-wide defect surrounded by five and ten reflection grating periods in the inner and outer sides, respectively. The inset of Fig. 2 shows a $L-L$ curve of the same device, indicating a threshold at $P_{\text {th }}=1.0 \mathrm{~mW}$. Other devices exhibited even lower threshold levels, the lowest being $0.6 \mathrm{~mW}$.

At threshold, emission lines spaced approximately $14 \mathrm{~nm}$ apart appeared in the spectrum. At twice the threshold level, two additional emission lines appeared at $\lambda=1.608 \mu \mathrm{m}$ and $\lambda=1.623 \mu \mathrm{m}$. At $P_{\text {pump }}=2.4 \mathrm{~mW}$ three additional emission lines appeared at $\lambda=1.593 \mu \mathrm{m}, \lambda=1.612 \mu \mathrm{m}$, and $\lambda=1.626 \mu \mathrm{m}$. Increasing the pump even further resulted only in variation of relative intensities of the emitted modes.

To understand the spectral characteristics, we used a finite-difference time domain simulation tool to model the device. ${ }^{16}$ Figure 3 shows a comparison between the A, measured and the B, calculated spectra. Good agreement was found between the measured and the calculated spectra not only for the resonance

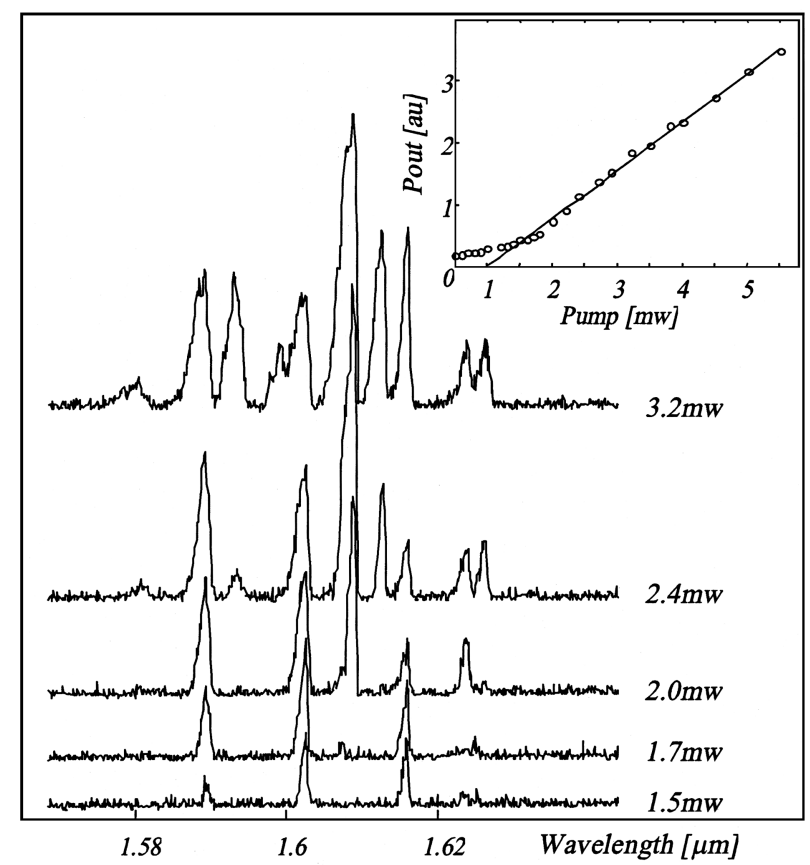

Fig. 2. Evolution of the emitted spectrum from the ABR shown in Fig. 1A as a function of pump intensity. Inset, $L-L$ curve of the device.

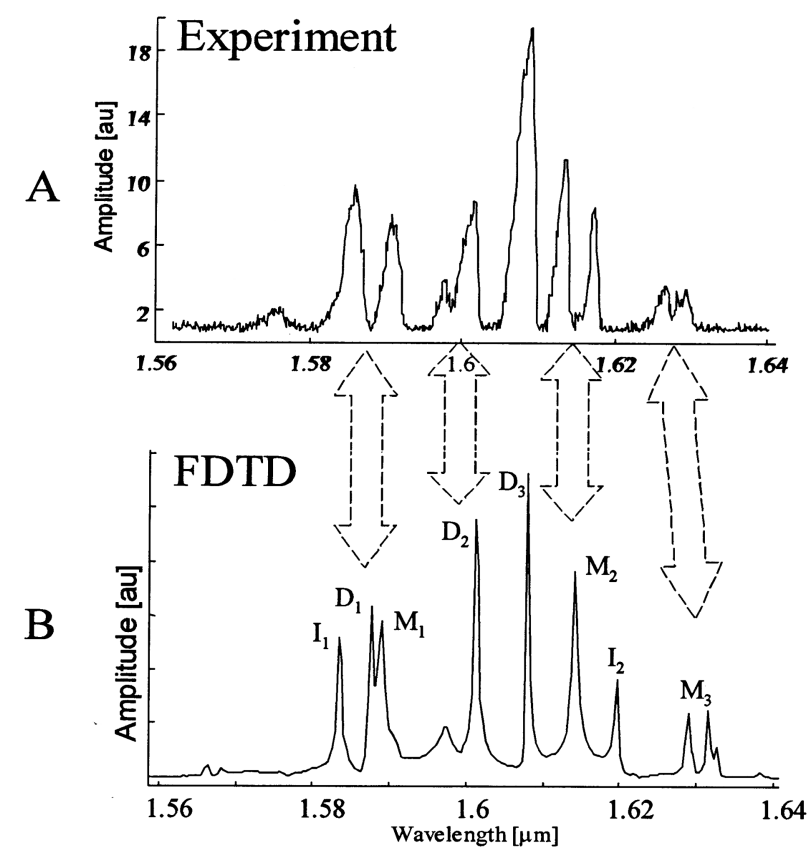

Fig. 3. A, measured and B, calculated spectral responses of the ABR shown in Fig. 1A. The D modes are confined in the defect; the I modes are confined in one of the rings of the internal reflector; the $M$ modes are not confined to a single ring. FDTD, finite-difference time domain. 


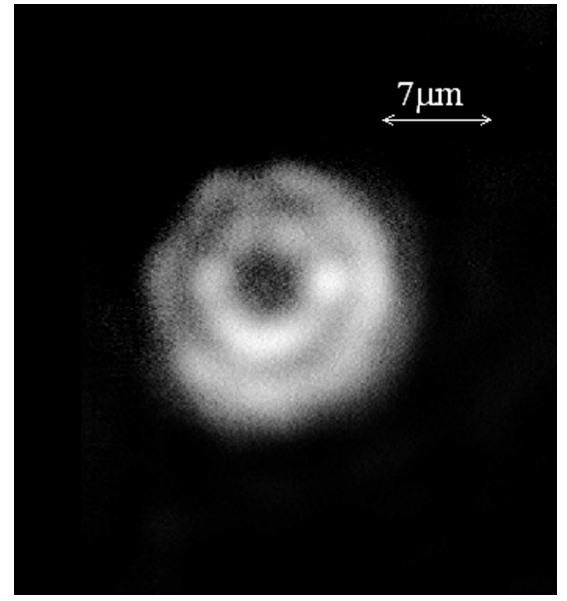

Fig. 4. IR image of the vertical emission from an ABR. The lasing pattern corresponds to mode $\mathrm{M}_{2}$.

wavelengths but also for the relative amplitudes. It should be noted that the measured spectrum is to some extent compressed compared with the calculated one. This is because the finite-difference time-domain model does not account for the material dispersion of the membrane. The field profiles of the various modes can be classified into three distinct categories (see Fig. 3A). The modes belonging to class D are confined within the defect with different angular propagation coefficients. The modes belonging to class I are confined in the ring closest to the defect from the internal side. These modes are actually guided by total internal reflection and are supported by the structure because of the use of second-order high-index layers. The rest of the modes, labeled M, are not localized in a specific layer but are distributed over several grating periods, peaking both in the defect and in one of the rings of the internal reflector. Unlike the I class, the M family modes are confined by Bragg reflection. The angular propagation factors of $\mathrm{M}_{1}$ and $\mathrm{M}_{2}$ are 41 and 31, respectively, corresponding to effective indices of 1.48 and 1.12 , defined by $n_{\text {eff }}=m \lambda / 2 \pi \rho_{\text {eff }}$, where $\lambda$ and $\rho_{\text {eff }}$ are, respectively, the resonance wavelength and the effective radius of the mode. These effective indices are lower than the refractive index of the adhesive filling the gaps, which confirms that the radial confinement mechanism is indeed Bragg reflection.

Figure 4 shows the intensity pattern emitted from the device at a pump level of $1.6 \mathrm{~mW}$. The emitted pattern consisted of two concentric and relatively wide rings of light. The radius of the outer ring corresponds to the radius of the defect, indicating that the lasing pattern belongs to the M family.
In summary, we have demonstrated annular Bragg lasers in a thin membrane of InGaAsP active semiconductor material. Lasing was achieved at room temperature under pulsed optical pumping conditions. Submilliwatt threshold levels were observed for ABRs with 7-8- $\mu \mathrm{m}$ defect radii pumped by a $30-\mu \mathrm{m}$-diameter spot. Imaging the vertical IR emission from the devices indicated localization of the field within the defect.

The authors thank Axel Scherer and Oskar Painter for access to fabrication facilities and George Paloczi and Reginald Lee for fruitful discussions. This work was supported by the National Science Foundation, the Defense Advanced Research Projects Agency, and the U.S. Air Force Office of Scientific Research. J. Scheuer's e-mail address is koby@caltech.edu.

\section{References}

1. A. Yariv, IEEE Photon. Technol. Lett. 14, 483 (2002).

2. B. E. Little, T. Chu, and H. A. Haus, Opt. Lett. 23, 1570 (1998).

3. C. K. Madsen and J. H. Zhao, Optical Filter Design and Analysis: a Signal Processing Approach (WileyInterscience, New York, 1999).

4. C. Y. Chao and L. J. Guo, Appl. Phys. Lett. 83, 1527 (2003).

5. K. J. Vahala, Nature 424, 839 (2003).

6. Y. Akahane, T. Asano, B. S. Song, and S. Noda, Nature 425, 944 (2003).

7. J. Scheuer and A. Yariv, Opt. Lett. 28, 1528 (2003).

8. J. Scheuer and A. Yariv, J. Opt. Soc. Am. B 20, 2285 (2003).

9. J. Scheuer, W. Green, G. DeRose, and A. Yariv, Proc. SPIE 5333, 183 (2004).

10. D. Labilloy, H. Benisty, C. Weisbuch, T. F. Krauss, C. J. M. Smith, R. Houdre, and U. Oesterle, Appl. Phys. Lett. 73, 1314 (1998).

11. A. Shaw, B. Roycroft, J. Hagarty, D. Labilloy, H. Benisty, C. Weisbuch, T. F. Krauss, C. J. M. Smith, R. Stanley, R. Houdre, and U. Oesterle, Appl. Phys. Lett. 75, 3051 (1999).

12. J. Scheuer and A. Yariv, IEEE J. Quantum Electron. 39, 1555 (2003).

13. W. M. J. Green, J. Scheuer, G. DeRose, and A. Yariv, Appl. Phys. Lett. 85, 3669 (2004).

14. L. A. Coldren and S. W. Corzine, Diode Lasers and Photonic Integrated Circuits (Wiley-Interscience, New York, 1995).

15. S. H. Kim, H. Y. Ryu, H. G. Park, G. H. Kim, Y. S. Choi, Y. H. Lee, and J. S. Kim, Appl. Phys. Lett. 81, 2499 (2002).

16. K. S. Yee, IEEE Trans. Antennas Propag. AP-14, 302 (1966). 